\title{
The Era of Massive Population III Stars: Cosmological Implications and Self\#Termination
}

\section{Citation}

Yoshida, Naoki, Volker Bromm, and Lars Hernquist. 2004. "The Era of Massive Population III Stars: Cosmological Implications and Self\#Termination." The Astrophysical Journal 605 (2): 57990. https://doi.org/10.1086/382499.

\section{Permanent link}

http://nrs.harvard.edu/urn-3:HUL.InstRepos:41381771

\section{Terms of Use}

This article was downloaded from Harvard University's DASH repository, and is made available under the terms and conditions applicable to Other Posted Material, as set forth at http:// nrs.harvard.edu/urn-3:HUL.InstRepos:dash.current.terms-of-use\#LAA

\section{Share Your Story}

The Harvard community has made this article openly available.

Please share how this access benefits you. Submit a story.

Accessibility 
To appear in ApJ April 20 ISsue, 2004

Preprint typeset using $\mathrm{LAT}_{\mathrm{E} X}$ style emulateapj v. 04/03/99

\title{
THE ERA OF MASSIVE POPULATION III STARS: COSMOLOGICAL IMPLICATIONS AND SELF-TERMINATION
}

\author{
NAOKI Yoshida ${ }^{1}$, Volker Bromm, And Lars Hernquist \\ Harvard-Smithsonian Center for Astrophysics, 60 Garden Street, Cambridge, MA 02138; \\ nyoshida@cfa.harvard.edu,vbromm@cfa.harvard.edu, lars@cfa.harvard.edu
}

To appear in ApJ April 20 issue, 2004

\begin{abstract}
The birth and death of the first generation of stars have important implications for the thermal state and chemical properties of the intergalactic medium (IGM) in the early universe. Sometime after recombination, the neutral, chemically pristine gas was reionized by ultraviolet photons emitted from the first stars, but also enriched with heavy elements when these stars ended their lives as energetic supernovae. Using the results from previous high-resolution cosmological simulations of early structure formation that include radiative transfer, we show that a significant volume fraction of the IGM can be metal-polluted, as well as ionized, by massive Population III stars formed in small-mass $\left(\sim 10^{6}-10^{7} M_{\odot}\right)$ halos early on. If most of the early generation stars die as pair-instability supernovae with energies up to $\sim 10^{53}$ ergs, the volume-averaged mean metallicity will quickly reach $Z \sim 10^{-4} Z_{\odot}$ by a redshift of $\sim 15-20$, possibly causing a prompt transition to the formation of a stellar population that is dominated by low-mass stars. In this scenario, the early chemical enrichment history should closely trace the reionization history of the IGM, and the end of the Population III era is marked by the completion of reionization and pre-enrichment by $z \sim 15$. We conclude that, while the pre-enrichment may partially account for the "metallicity-floor" in high-redshift Lyman- $\alpha$ clouds, it does not significantly affect the elemental abundance in the intracluster medium.

Subject headings: cosmology: theory - galaxies: formation — intergalactic medium — stars: formation
\end{abstract}

\section{INTRODUCTION}

Chemical elements heavier than lithium are thought to be produced exclusively through stellar nucleosynthesis. The primordial cosmic gas remains chemically pristine until the first supernova (SN) explosions expel metals that are produced in the precursor stars. High-redshift observations of the Lyman- $\alpha$ forest (e.g., Songaila 2001; Pettini et al. 2003), damped Lyman- $\alpha$ systems (e.g., Prochaska 2002), and quasars (e.g., Freudling, Corbin, \& Korista 2003) all indicate that heavy elements are distributed in various cosmic environments by $z \sim 5$. While the actual transport mechanism and the nature of the primary sources have not been determined, it is often suggested that the origin of these heavy elements may be attributed to the first generation of stars, the so-called Population III stars (e.g., Ostriker \& Gnedin 1996; Madau, Ferrara, \& Rees 2001; Thacker, Scannapieco, \& Davis 2002; Qian, Sargent, \& Wasserburg 2002).

In the standard cosmological models based on cold dark matter (CDM), the first cosmological objects are predicted to form at redshifts greater than 20 (e.g., Couchman \& Rees 1986; Tegmark et al. 1997; Abel et al. 1998; Yoshida et al. 2003a). Thus, the first heavy elements are likely to have been processed at such early epochs. Intriguingly, the recent measurement of a large Thomson optical depth by the Wilkinson Microwave Anisotropy Probe (WMAP) satellite (Kogut et al. 2003; Spergel et al. 2003) provides evidence that the universe was reionized very early on, supporting the above notion that the first stars could form at $z \gtrsim 20$ (e.g., Cen 2003; Haiman \& Holder 2003; Wyithe \& Loeb 2003a,b; Sokasian et al. 2003a).
The relative abundances of heavy elements produced in the first stars are of great importance because observations of the elemental abundance pattern of ultra metalpoor stars (e.g., Christlieb et al. 2002) can place strong constraints on the environments in which these stars were formed and possibly on the progenitor mass (Umeda \& Nomoto 2003; Schneider et al. 2003a). Theoretical modeling of the formation of the first stars (Abel, Bryan, \& Norman 2002; Bromm, Coppi, \& Larson 2002; Omukai \& Palla 2003) consistently indicate that they were rather massive, with characteristic mass being possibly several hundred solar masses. If the first stars are indeed as massive as $\sim 200 M_{\odot}$, they end their lives as energetic SNe via the pair-instability mechanism (e.g., Barkat, Rakavy, \& Sack 1967; Bond, Arnett, \& Carr 1984; Fryer, Woosley, \& Heger 2001; Heger \& Woosley 2002; Woosley, Heger, \& Weaver 2002 ), releasing a total energy of up to $\sim 10^{53}$ ergs. Such energetic explosions in the early universe are violently destructive: they expel the ambient gas out of the gravitational potential well of small-mass dark matter halos, causing an almost complete evacuation (Bromm, Yoshida, \& Hernquist 2003, hereafter Paper I; Wada \& Venkatesan 2003).

Since the massive stars process a substantial fraction of their mass into heavy elements, early SN explosions may provide an efficient mechanism to pollute the surrounding intergalactic medium (IGM). Models of stellar evolution (e.g., Heger \& Woosley 2002) predict that, in massive zero-metallicity stars, up to $\sim 40-50 \%$ of the total stellar mass is processed into heavy elements in the core and, in the pair-instability case, all of it is finally ejected during the explosion without leaving any compact remnant

\footnotetext{
${ }^{1}$ National Astronomical Observatory of Japan, Mitaka, Tokyo 181-8588, Japan.
} 
behind. Blast waves triggered by the $\mathrm{SN}$ explosions will then drive supersonic flows of gas that are enriched with the processed heavy elements. Consequently, because of the short lifetimes of such massive stars, the emergence of the early generation stars should be almost immediately followed by metal enrichment of the IGM; i.e., prompt metal enrichment could be achieved efficiently by Population III SNe in the early universe.

The transport of metals by SN-driven winds has been studied extensively in the context of conventional galaxy formation (e.g., Larson 1974; Dekel \& Silk 1986; Vader 1986; Mac Low \& Ferrara 1999; Aguirre et al. 2001a,b; Madau et al. 2001; Scannapieco, Thacker, \& Broadhurst 2001; Mori, Ferrara, \& Madau 2002; Furlanetto \& Loeb 2003; Springel \& Hernquist 2003a,b). In fact, gas flows induced by SN explosions appear to be the most plausible way of distributing metals, which are observed to exist under a broad range of environments. Interestingly, an array of observations indicate that the metals were produced and dispersed at early stages of structure formation. Spectra of distant quasars consistently show that there are at least some metals in the diffuse IGM at $z \simeq 2-3$ (e.g., Schaye et al. 2000, 2003). Qian \& Wasserburg $(2001,2002)$ argue that the observed abundance patterns of metal-poor stars can be explained by the "prompt-inventory" of heavy elements from very massive stars. Loewenstein (2001) points out that a contribution from very massive metalfree stars may account for the observed abundance anomalies of oxygen, silicon, and iron in the intracluster medium (ICM). More recently, Freudling et al. (2003) discovered a strong Fe II emission feature from a $z \simeq 6$ quasar, providing strong evidence that iron is produced in some stars/galaxies already at $z \gtrsim 10$, which is expected if black hole growth is regulated by star formation (e.g., Di Matteo et al. 2003a,b). Together with the implication of an early reionization derived from the WMAP results, these observations indicate that Population III stars may have been an important contributor of heavy elements in the diffuse IGM/ICM as well as the source of radiation responsible for the early reionization of the universe. The association of the IGM metal enrichment with the UV-photon production from the first stars was previously discussed by Oh et al. (2001), and more recently by Stiavelli, Fall, \& Panagia (2003). In these studies, however, the analysis was not based on a detailed modeling of the high-redshift star formation rate, and the precise epochs of Population III pre-enrichment and reionization therefore remained somewhat uncertain.

In the present paper, we revisit the cosmic metal production in the context of early structure formation. We explore the possibility that the first stars are responsible for reionization of the universe at $z \gtrsim 15$, while also causing a significant, prompt chemical enrichment of the IGM. We argue that, if the early reionization was the result of very massive Population III stars, the mean metallicity should reach a critical level at which the efficiency of gas cooling is greatly enhanced, thus changing the overall mode of star formation (e.g., Mackey, Bromm, \& Hernquist 2003).

The hydrodynamic transport of heavy elements induced by SN explosions is studied numerically in Paper I, using high-resolution, three-dimensional cosmological simulations. We show that a large amount of the gas is ex- pelled out of the shallow gravitational potential of the host "minihalos". The simulations in Paper I further demonstrate that, if the explosion kinetic energy is as high as $\sim 10^{53}$ ergs, nearly $90 \%$ of the pre-enriched gas in the immediate vicinity of the explosion site eventually escapes out of the host halo, reaching up to a distance of $\sim 0.5 \mathrm{kpc}$ in a few million years. Since multiple $\mathrm{SNe}$ are expected to be occurring at $z \gtrsim 15$ in distant star-forming regions within a cosmological volume, the total amount of metals produced and distributed into the IGM may well exceed the critical level required to affect the thermal and chemical properties of the IGM. The transition in stellar populations, from Population III to Population II, may then be naturally caused early on.

Throughout the present paper, except in section ?? where we consider a variant CDM model, we work with a flat $\Lambda$-dominated Cold Dark Matter universe with matter density $\Omega_{\mathrm{m}}=0.3$, cosmological constant $\Omega_{\Lambda}=0.7$ and the Hubble constant at the present time $h=0.7$ in units of $100 \mathrm{~km} \mathrm{~s}^{-1} \mathrm{Mpc}^{-1}$. We set the baryon density to $\Omega_{\mathrm{b}}=0.04$ and the initial density fluctuations are normalized to $\sigma_{8}=0.9$. These parameters are consistent with those employed in the numerical simulations we refer to in the following sections.

\section{FEEDBACK FROM EARLY GENERATION STARS}

In this section we summarize various feedback effects from the first stars, and derive useful order-of-magnitude estimates for quantities needed to study these effects. The ultimate fate of a metal-free star depends critically on its mass. Theoretical stellar models predict the following classification (Heger \& Woosley 2002; Heger et al. 2003): (1) $8<M_{*}<25 M_{\odot}$ : these stars explode as core-collapse $\mathrm{SNe}$ and leave a neutron star behind, (2) $25<M_{*}<40 M_{\odot}$ : these explode as faint Type II SNe and leave black holes behind after fall-back of most of the envelope (see Umeda \& Nomoto 2002, 2003), (3) $40<M_{*}<140 M_{\odot}$ : these do not explode as $\mathrm{SNe}$ and directly collapse into black holes, (4) $100<M_{*}<140 M_{\odot}$ : these experience a pulsational instability and eject their outer envelope, again leaving a black hole behind, (5) $140<M_{*}<260 M_{\odot}$ : these explode as pair-instability $\mathrm{SNe}$, causing complete disruption, and (6) $M_{*}>260 M_{\odot}$ : these collapse, in the absense of rotation, directly into massive black holes.

In summary, only the pair-instability case can substantially contribute to the metal enrichment of the IGM, whereas remnant black holes (or neutron stars) are left in the other cases, which we shall discuss in $\S 2.5$. While early generation stars may be born with a broad range of masses (e.g., Bromm, Coppi, \& Larson 1999; Nakamura \& Umemura 2001, 2002; Omukai \& Yoshii 2003), very massive stars are dominant in causing various feedback effects to the IGM in the early universe such as ionization, heating, and chemical enrichment. Thus, throughout the present paper, we primarily consider very massive $\left(\sim 100-300 M_{\odot}\right)$ metal-free stars as Population III stars.

\subsection{Population III Stars as Reionization Sources}

We first discuss radiative feedback. The efficiency of ionizing photon production from massive Population III stars is obtained from detailed calculations (e.g., Bromm, Kudritzki, \& Loeb 2001b; Schaerer 2002, 2003). As our fiducial case, we consider a metal-free star with mass 
$M_{*}=200 M_{\odot}$. Such a star emits about $3 \times 10^{50}$ ionizing photons per second, and has a main-sequence lifetime of $\sim 2$ million years.

Consider a population of such massive stars formed in a cubic comoving-Mpc volume by $z$. We introduce a quantity

$$
q(z) \equiv \frac{N_{\mathrm{ph}}(z)}{N_{\mathrm{H}}},
$$

where $N_{\mathrm{ph}}(z)$ is the cumulative number of ionizing photons emitted from the stars, and $N_{\mathrm{H}}=4 \times 10^{66}$ is the number of hydrogen atoms in the volume. This 'number of photons per atom' $q(z)$ serves as an indicator of the global ionization fraction as a function of $z$. Clearly, in order to completely ionize the IGM, at least the same number of UV photons as the number of hydrogen atoms must be emitted within the volume. It follows from these numbers that more than a few hundred very massive stars must be formed in a comoving-Mpc volume to cause complete reionization.

\subsection{Population III Stars as Sources of Heavy Elements}

Massive metal-free stars are not only luminous ionizing sources, but also efficient factories of heavy elements. For stars with masses $\sim 100-300 M_{\odot}$, typically up to $\sim 50 \%$ of the mass is processed into heavy elements in the core, consisting mostly of carbon, oxygen, silicon and iron. We denote this mass fraction with $f_{Z}$, and set it to be 0.5 as our fiducial value for massive stars in the remainder of the paper. We further introduce a parameter

$$
\zeta=\frac{N_{\mathrm{n}}}{N_{\gamma}}
$$

where $N_{\mathrm{n}}$ is the total number of nuclei in the heavy elements processed in the core, and $N_{\gamma}$ is the total number of ionizing photons emitted by the star during its lifetime. Namely, with $m_{\mathrm{p}}$ denoting the proton mass, $m_{\mathrm{p}} \times N_{\mathrm{n}}$ is the total mass of metals produced in the star. This quantity gives the ratio of the stellar metal to photon production which is, in principle, uniquely determined by the structure and evolution of the respective star. For a metal-free star with mass $200 M_{\odot}$ that is destined to die in a pairinstability supernova, the actual value is approximately

$$
\zeta_{\mathrm{PISN}}=\frac{f_{Z} \times 200 M_{\odot} / m_{p}}{2 \times 10^{64}}=\frac{10^{59}}{2 \times 10^{64}} \sim 5 \times 10^{-6},
$$

for $f_{Z}=0.5$. It is straightforward to compute the value for $\zeta$ for less massive stars or for stars with finite metallicity from stellar evolution models.

Intriguingly, this parameter $\zeta_{\mathrm{PISN}}$ for the very massive stars that are destined to explode as pair-instability $\mathrm{SNe}$ can be expressed in terms of fundamental physical constants as follows. We estimate the total number of ionizing photons produced during the lifetime of a Population III star with mass $M_{*}$ as $N_{\gamma} \simeq 0.007 M_{*} c^{2} / \epsilon_{\text {ion }}$. Here, $\epsilon_{\text {ion }} \simeq e^{2} / a_{0} \simeq \alpha^{2} m_{e} c^{2}$ is the binding energy of an electron in an $\mathrm{H}$ atom, with $a_{0}$ being the Bohr radius, $\alpha \simeq 1 / 137$ the fine-structure constant, and $m_{e}$ the electron rest mass. Assuming the limiting case of a $100 \%$ nuclear burning efficiency, we can write for the total number of nucleons processed into heavy elements: $N_{n} \simeq M_{*} / m_{p}$. We now have, for very massive stars that die as pair-instability SNe,

$$
\zeta_{\mathrm{PISN}}=\frac{\alpha^{2} m_{e}}{\alpha_{\mathrm{pn}}^{2} m_{p}} \sim 4 \times 10^{-6},
$$

where $\alpha_{\mathrm{pn}}^{2} \simeq 0.007$ describes the strength of the pionnucleon interaction. The production of ionizing photons and metals in a massive star is thus determined by the ratio of the atomic and nuclear binding energies.

\subsection{Population III Supernova Explosions}

The processed metals can be distributed into the IGM only if some transport mechanism operates, otherwise the metals would be confined within a small region and the diffuse IGM would be largely unaffected. In this section, we first describe the hydrodynamics of a single Population III SN explosion in the early universe and then show that the gas and the heavy elements can be transported over a large distance.

Assuming spherical symmetry of the system, we apply the usual Sedov-Taylor self-similar analysis for a given explosion energy and density of the ambient medium. We first need to specify the density profile of the surrounding IGM in order to fully describe the evolution of the SN blast wave. For a density profile approximated by a simple power-law, $\rho \propto r^{-w}$, the expansion of a blast wave is described by the self-similar solution:

$$
R_{\mathrm{s}}=\xi\left(\frac{E t^{2}}{n}\right)^{1 /(5-w)},
$$

where $\xi$ is a constant determined by the energy integral, $E$ is the explosion energy, $n$ the particle number density of the surrounding medium, and $t$ the time elapsed since the explosion (Sedov 1959; see also Ostriker \& McKee 1988). In reality, the gas density profile can be more complex because photo-evaporation due to radiation from the central star is very effective in small halos $\left(T_{\text {vir }} \lesssim 10^{4} \mathrm{~K}\right)$. Large pressure gradients in the ionized region drive an outward gas flow before the explosion is triggered (see Paper I). Hence, although initially the gas density is high around the star, it is expected to be substantially reduced by the time when the star explodes, and the density profile will be much shallower except in the outer edge where a supersonic shockwave is propagating. Taking the effective photo-evaporation into account, we assume the density of the ambient medium around the explosion site is approximately constant at the mean IGM density at $z$,

$$
n_{\mathrm{H}, \mathrm{IGM}} \simeq 0.0014\left(\frac{\Omega_{\mathrm{b}} h^{2}}{0.02}\right)\left(\frac{1+z}{20}\right)^{3},
$$

where $\Omega_{\mathrm{b}}$ is the baryon density and $h$ is the Hubble constant in units of $100 \mathrm{~km} \mathrm{~s}^{-1} \mathrm{Mpc}^{-1}$. Note that the shock radius in equation (51) scales only weakly with the density and the explosion energy unless the density profile is very steep. The three-dimensional simulations in Paper I show that the evolution of the shock radius is well described by equation (15) in a piecewise manner for regions having density profiles with different slopes. For a constant density medium, the Sedov-Taylor self-similar solution reduces to

$$
R_{\mathrm{s}} \simeq 200\left(\frac{E_{51}}{n_{0}}\right)^{1 / 5} t_{7}^{2 / 5} \mathrm{pc}
$$


where $R_{\mathrm{S}}$ is the shock radius, $E_{51}$ the total SN energy in units of $10^{51} \mathrm{ergs}, n_{0}$ the particle number density of the ambient medium in units of $1 \mathrm{~cm}^{-3}$, and $t_{7}$ the elapsed time since the explosion in units of $10^{7}$ years. For the explosion energy, Heger \& Woosley (2002) calculate the energy released by a Population III pair-instability SN to be in the range $\sim 10^{51}-10^{53}$ ergs.

The above solution is valid if the gas remains adiabatic, but breaks down when the shock becomes radiative. The hot gas in the interior of high redshift $(z \gtrsim 10)$ SN remnants (SNRs) cools partly via inverse Compton cooling. The characteristic cooling time for this process is given by

$$
t_{\text {Comp }} \simeq 10^{7}\left(\frac{1+z}{20}\right)^{-4} \mathrm{yr} .
$$

Note the strong dependence on redshift. At $z=20$, the SNR will remain approximately adiabatic (hence equation [7] is valid) until $\sim 10^{7}$ years after the explosion. However, this provides only a maximum timescale. In the postshock region, the gas density and temperature are so high that hydrogen and helium atomic line cooling is the dominant cooling process. Thus, a thin dense shell could be formed faster than the above timescale. The exact time when the post-shock region becomes radiative depends on the total explosion energy and the density of the ambient medium in a complicated manner. The shell forms when the local cooling time $t_{\text {cool }}(r)$ at radius $r$ becomes shorter than the expansion timescale $R_{\mathrm{s}} / \dot{R}_{\mathrm{s}}$. We use the analytic Sedov-Taylor solution for the temperature $T(r)$ and density $\rho(r)$ of the gas to compute the local gas cooling rate $\Lambda(\rho(r), T(r))$, assuming an explosion energy $E=10^{53}$ ergs and the gas density $n=0.0014 \mathrm{~cm}^{-3}(\sim$ the mean IGM density at $z \sim 20$ ). Recent numerical simulations by Whalen, Abel, \& Norman (2003) indeed show that photoevaporation by the central star reduces the final gas density within the host halo to very small values, comparable to the mean IGM density. In this case, the shell forms at a time $\sim 3 \times 10^{6} \mathrm{yr}$. Then the shell radius (the "stallingradius") is $\sim 1.2 \mathrm{kpc}$, and it expands only slowly during the later snow-plow stage as $R_{\mathrm{s}} \propto t^{1 / 4}$ (see Figure 1 in Paper I). This is perhaps an optimistic estimate because the gas evacuation efficiency by radiation from the stars depends on the host halo mass and the gas density profile. For a ten times higher density, $n=0.014 \mathrm{~cm}^{-3}$, the stalling-radius is calculated to be only $40 \%$ smaller, $\sim 0.7$ $\mathrm{kpc}$, which is more consistent with the result of our simulation presented in Paper I. Overall, because of the weak dependence of the self-similar solution (equation [7) on the density, the above estimates will not be significantly affected by (possible) variations in the gas density around the $\mathrm{SN}$ sites.

The momentum-driven slow expansion is still important over a cosmological timescale (Bertschinger 1983), with the radius doubling every twenty folds in time. Thus, if mixing of the original ejecta with the ambient medium is efficient, the expelled metals can travel over a large distance $(\sim$ a few kpc) within a Hubble time. Three-dimensional numerical simulations of Nakasato \& Shigeyama (2000) show that the SN ejecta are fairly well mixed within the remnant, with the enriched volume fraction being close to unity. Wada \& Venkatesan (2003) show that metal-dispersal is very efficient even in dense, disk- shaped gas clouds. Therefore, the processed metals are likely to be transported as far as the shell radius.

\subsection{Global Quantities}

Let us calculate the evolution of the volume-averaged mean metallicity of the IGM. We allow the uncertainty associated with the characteristic mass, or initial mass function (IMF), of Population III stars by assuming that a fraction, $f_{\mathrm{PISN}}$, of the Population III stars have masses in the pair-instability range. Suppose that $n_{*}(z)$ stars are formed by $z$ in a unit comoving volume. The total mass of metals produced within the volume is then

$$
M_{Z}=f_{\mathrm{PISN}} \times n_{*}(z) f_{Z} M_{*} .
$$

We further normalize this quantity to the mass of the IGM within the volume, to obtain the mean metallicity:

$$
\begin{aligned}
\bar{Z} & =\frac{M_{Z}}{M_{\mathrm{IGM}}}=\frac{n_{*}(z) \times f_{\mathrm{PISN}} \times f_{Z} M_{*}}{m_{\mathrm{p}} \times N_{\mathrm{H}}} \\
& \approx f_{\mathrm{PISN}} \times q(z) \times \zeta_{\mathrm{PISN}} .
\end{aligned}
$$

Recall that $\zeta_{\text {PISN }}$ is approximately a constant for massive metal-free stars that explode as pair-instability $\mathrm{SNe}$ (equation [3), and $q(z)$ traces the IGM ionization fraction. The above equation simply shows that the total quantity of heavy elements ejected into the IGM is proportional (weighted by the fraction $f_{\mathrm{PISN}}$ ) to the number of $\mathrm{UV}$ photons emitted by $z$.

We now consider the simplest case where all of the Population III stars have a mass of $200 M_{\odot}$ and explode as pair-instability $\mathrm{SN}$, i.e., $f_{\mathrm{PISN}}=1$. We further assume that $q_{\text {reion }} \sim 2$ marks the epoch of reionization (see, e.g., fig. 8 of Sokasian et al. 2003b). Setting $\zeta_{\mathrm{PISN}}=5 \times 10^{-6}$, we obtain

$$
\left.\bar{Z}\right|_{z_{\text {reion }}}=10^{-5} \approx 5 \times 10^{-4} Z_{\odot},
$$

which coincides with the critical metallicity necessary for the formation of low-mass gas clumps (Bromm et al. 2001a; see also Mackey et al. 2003). Note also that very massive stars become progressively unstable for increasing metallicity (Baraffe, Heger, \& Woosley 2001). Therefore, for $f_{\mathrm{PISN}}=1$, the epoch when the transition in the mode of star formation occurs should closely match the first reionization epoch. Note, however, that we have neglected recombination in the above argument. If Population III star formation occurs rather gradually, taking a considerably longer time than a recombination time, most of the UV photons are simply consumed by recombination without increasing the global ionization fraction of the IGM. Approximating the IGM density with the cosmic mean density, we estimate the recombination timescale for hydrogen:

$$
t_{\mathrm{rec}} \approx 10^{8} \mathrm{yr}\left(\frac{1+z}{20}\right)^{-3}
$$

If the increase in the number of UV photons is sufficiently rapid, compared to $t_{\text {rec }}$, then the ionization fraction of the IGM should monotonically increase. This is indeed the case for the model we describe below.

An important question is when and how rapidly did the early generation stars emerge? We address this issue using the formation rate of Population III stars derived by Yoshida et al. (2003a). Their result is based 
on a semi-analytic model coupled with the outputs of a large cosmological $N$-body simulation. In their model, star-forming regions are identified as halos in which the gas has been able to cool via molecular hydrogen cooling, and the usual 'one-star-per-halo' assumption is made such that only a single star is formed per star-forming gas cloud. The model also includes the negative feedback owing to photo-dissociation of molecular hydrogen by photons in the Lyman-Werner band. Hence, the model provides a conservative estimate for the early star-formation rate (SFR). It is worth noting that the resulting SFR was found to be in good agreement with that obtained from the hydrodynamic simulations of Sokasian et al. (2003a) which include radiative transfer as well as various feedback processes. We revised the empirical fit to the SFR in Yoshida et al. (2003a) and found that it is well-described by a particular form proposed by Hernquist \& Springel (2003):

$$
\dot{\rho}_{*}(z)=\dot{\rho}_{*, 0} \frac{\chi^{p}}{1+\tilde{\alpha}(\chi-1)^{\tilde{q}} \exp \left(\beta \chi^{r}\right)},
$$

where

$$
\chi(z) \equiv\left(\frac{H(z)}{H_{0}}\right)^{2 / 3}
$$

and, in this case, $p=5, \tilde{q}=1, r=1.85, \tilde{\alpha}=0.01$, $\beta=0.04, \dot{\rho}_{*, 0}=3.0 \times 10^{-8} M_{\odot} \mathrm{yr}^{-1} \mathrm{Mpc}^{-3}$.

Figure 1 shows the Population III SFR. The flattening at $z<25$ reflects the suppression of the gas cooling due to global radiative feedback, showing the self-regulating nature of early star formation (e.g., Ricotti, Gnedin, \& Shull 2002; Wise \& Abel 2003; Yoshida et al. 2003a). We compare the derived SFR with the result of the hydrodynamic simulations of Sokasian et al. (2003a). To this end, we have averaged over all the nine models considered in Sokasian et al. (2003a) which differ in the star-formation efficiency as well as the strengths of the feedback effects. Overall, the agreement between the semi-analytic prediction and the simulation results is quite good. As Sokasian et al. (2003a) note, the SFR derived from their simulations may suffer from finite boxsize effects at high redshifts $(z>25)$, which accounts for the small discrepancy between the analytic model and the simulation result seen in Figure 1 (see also Yoshida et al. 2003b; Barkana \& Loeb 2003)

In the middle panel of Figure 1, we plot the cumulative number of ionizing photons per baryon $q(z)$. We compute the number of ionizing photons assuming a single massive $\left(M_{*}=200 M_{\odot}\right)$ star emits ionizing photons at a rate of $3 \times 10^{50} \mathrm{~s}^{-1}$ during its main-sequence lifetime (Bromm et al. 2001b). The dashed line in the middle panel indicates $q=1$, which is achieved by $z=17$. We note that the total number of UV photons increases by more than an order of magnitude between $z=22$ and $z=17$. The corresponding time interval is $\sim 50 \mathrm{Myr}$, whereas the recombination timescale at $z \sim 20$ is $\sim 100 \mathrm{Myr}$. Therefore, to lowest order, the photon consumption due to hydrogen recombination can be neglected. In addition, since the photon escape fraction from minihalos is expected to be of order unity (Whalen et al. 2003), and the clumping of the IGM at these early epochs is small (Haiman, Abel \& Madau 1999), $q \sim$ a few should suffice to reionize a substantial fraction of the IGM (e.g., Sokasian et al. 2003b).
It is worth mentioning that, in our model, the time when $q=1$ coincides with the epoch of reionization suggested by the $W M A P$ data, $z=17 \pm 4$ (assuming an instantaneous reionization, see Kogut et al. 2003). At the same time, as shown in the bottom panel in Figure 1, the global mean metallicity reaches the metallicity floor of a few $\times 10^{-4} Z_{\odot}$. We emphasize that this global mean is merely an approximate estimate because, locally around the SN sites where the formation of second generation stars is likely to take place, the gas metallicity could be much higher than this value.
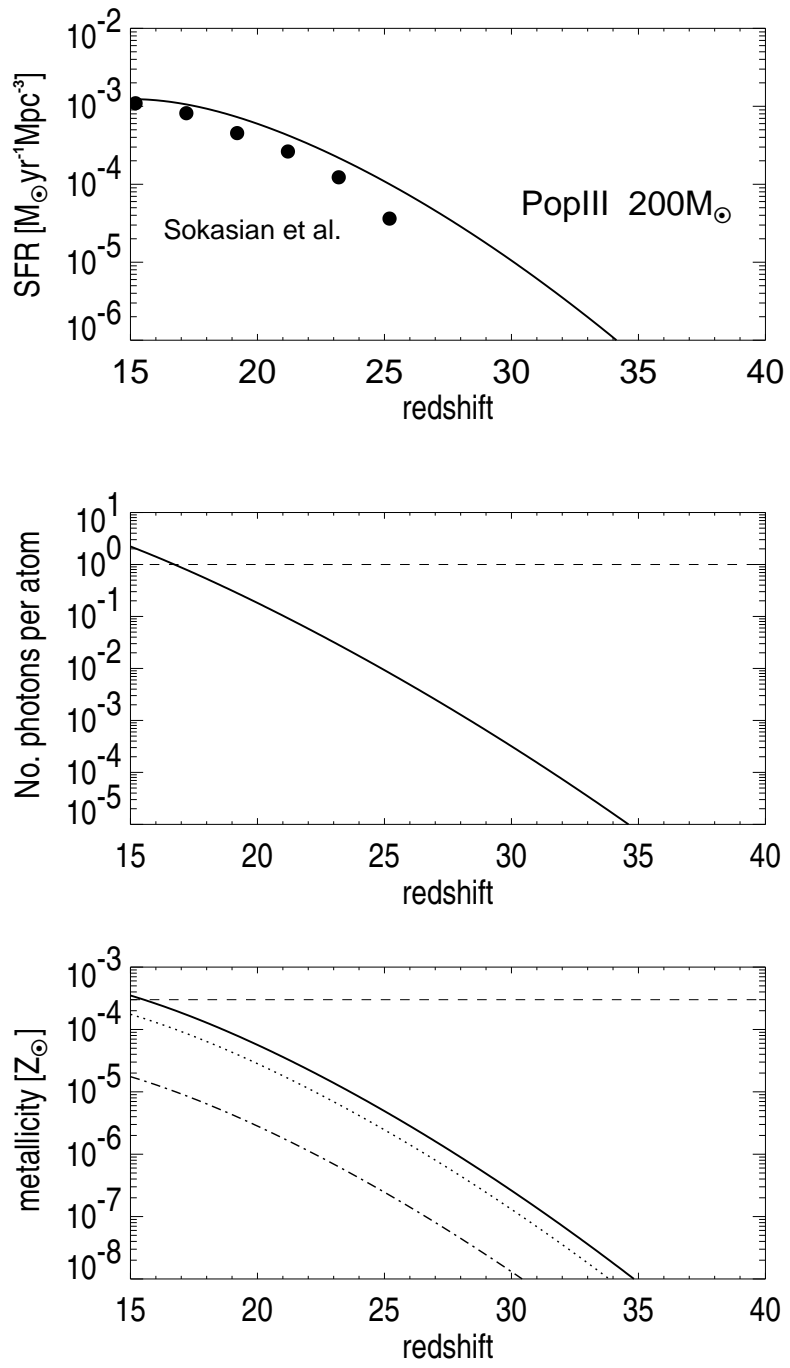

Fig. 1.- The global star formation rate density (top), the cumulative number of photons per hydrogen atom (middle), and the mean IGM metallicity $\bar{Z}$ (bottom), as functions of redshift. In the top panel we compare our semi-analytic prediction (solid line) with the result of numerical simulations of Sokasian et al. (2003a) (filled circles). The dashed line in the middle panel indicates $q=1$. The critical metallicity $10^{-3.5} Z_{\odot}$ is also indicated by the dashed line in the bottom panel. We plot three cases with mixing efficiency of the heavy elements with the diffuse IGM, 100\% (solid line), $50 \%$ (dotted line), and 5\% (dot-dashed line).

\subsection{Remnant Black Holes}

In the previous sections, we have exclusively considered very massive stars that explode as pair-instability $\mathrm{SNe}$, i.e., those with masses $140-260 M_{\odot}$. Stars with masses outside this range are likely to leave remnant black holes behind 
(Fryer et al. 2001). In particular, stars even more massive than $260 M_{\odot}$ completely collapse to black holes, which could be the seeds of supermassive black holes (Madau \& Rees 2001; Islam, Taylor, \& Silk 2003; Volonteri, Haardt, \& Madau 2003).

It is straightforward to compute the evolution of the mass density locked up in black holes using our model Population III SFR. Let us adopt the simplest model where all the Population III stars are extremely massive and become black holes. Although this is an implausible situation because then the IGM would remain chemically pristine forever and the "star-formation conundrum" would arise (see Schneider et al. 2002), it is illustrative to study the evolution of the black hole mass density in this scenario.

We consider three cases with $M_{*}=300,600,1000 M_{\odot}$, and the resulting mass density is plotted in Figure 2 Note that our model for the Population III star formation is based on the 'one-star-per-halo' assumption and thus the star formation rate density scales approximately with $M_{*}$. Figure 2]shows that the mass density at $z \sim 15$ is comparable to the present-day mass density in black holes in the local universe (e.g., Merritt \& Ferrarese 2001) for high mass values. Note that these Population III blackholes are likely to have sunk to the galactic centers by the present epoch due to dynamical friction (e.g., Madau \& Rees 2001).

Obviously, this extreme top-heavy mode of Population III star formation should be stopped at some point. Otherwise too much baryonic mass would be converted into black holes locally by $z=0$.

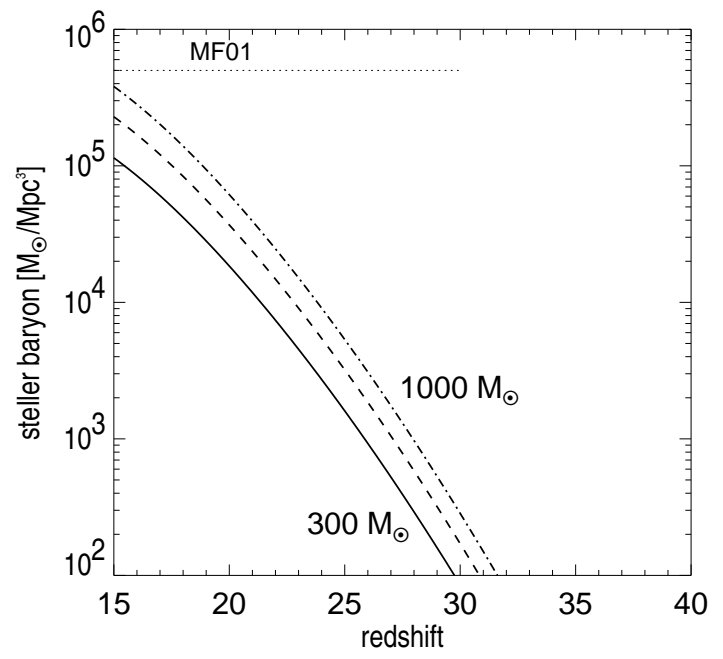

FIG. 2.- The total mass of baryons converted to stars per unit comoving volume, for $M_{*}=300$ (solid line), 600 (dashed line), and $1000 M_{\odot}$ (dot-dashed line). The horizontal dotted line indicates the present-day mass density in black holes in the local universe estimated by Merritt \& Ferrarese (2001).

\section{IMPLICATIONS}

\subsection{The End of the Population III Era}

One may naively expect that Population III star formation could continue down to moderate redshifts, $z \sim 5-10$. We argue, based on the results of our model, that either the completion of reionization or prompt enrichment to a level of $\bar{Z} \sim 10^{-4} Z_{\odot}$ by $z \sim 15$ should mark the end of the Population III era.

When a large fraction of the IGM is reionized, effec- tive photo-evaporation of minihalos by internal and external sources occurs (e.g., Shapiro, Iliev, \& Raga 2003), and virtually no gas can be trapped within low mass halos. Furthermore, SN explosions cause nearly complete evacuation of the host halos (see Paper I). Therefore, regardless of the efficiency of the proposed positive feedback on molecular hydrogen formation (e.g., Ferrara 1998; Oh 2001), it should take some time for the heated and expelled gas to cool and condense. Subsequent star formation can take place only after the gas falls back into the halos again. Accordingly, there should be an appreciable time gap between the first star formation and the onset of the second-generation star formation. We conservatively estimate this 'recuperation time' (Sokasian et al. 2003a) to be of the order of the dynamical time scale for the halos. The dynamical time scale is given by

$$
t_{\mathrm{dyn}}=\sqrt{\frac{3 \pi}{32 G \rho}},
$$

where $G$ is the gravitational constant and $\rho$ the total mass density of a halo. Assuming that the mass density of a halo (including dark matter) is 200 times the cosmic mean density, we compute the dynamical time for halos collapsing at $z=15$ to be $\sim 50 \mathrm{Myr}$. Since the cosmic time difference between $z=15-18$ (or $z=13-15$ ) is $\sim 60 \mathrm{Myr}$ for the standard $\Lambda \mathrm{CDM}$ model, star formation is likely to be quenched for at least a duration of $\Delta z \sim$ a few in redshift. It might seem that halos with virial temperature greater than $10^{4} \mathrm{~K}$ are unaffected by photo-heating due to reionization (e.g., Oh \& Haiman 2002). We point out that, in the hierarchical universe, all the massive halos used to be minihalos at earlier epochs. Hence, according to our model of Population III star-formation, the gas within them is likely to have been already expelled either by radiation from the Population III stars or by SN explosions. The situation would be slightly different in halos that are rapidly growing in mass because such halos may be able to trap the ionized hot gas. However, SN explosions with energies $\sim 10^{53}$ ergs are still able to cause an outward gas flow even in the large halos. If the feedback from the first massive star on its vicinity occurs by $\mathrm{z}=15$, as in our model, then the formation of the second-generation stars in the same region (i.e., protogalaxy) will begin at $z \lesssim 13$. Further, very massive stars are already pulsationally unstable when the metallicity is $\sim 10^{-4} Z_{\odot}$, even if they are formed (Baraffe et al. 2001). The minimum metallicity floor is established in all the star-forming regions by $z \sim 15$ if the first stars explode as pair-instability $\mathrm{SNe}$, thus causing a 'global' regulation of the star formation activity lasting until $z \sim 13$. Note that significant cosmic variance may be induced by large-scale $(\gg 1 \mathrm{Mpc})$ density fluctuations. The cosmic scatter in the number density of the star-forming clouds should then cause a substantial spread in the epoch of self-termination (e.g. Scannapieco, Schneider \& Ferrara 2003).

An alternative scenario for second-generation star formation is self-propagating star formation in the thin, dense shell formed by early SN explosions (e.g., Ostriker \& Cowie 1981; Wandel 1985; McCray \& Kafatos 1987). Such phenomena are indeed observed in the local universe (e.g., Dopita et al. 1985; Efremov \& Elmegreen 1998) and may have taken place in the early universe as well (Mackey et 
al. 2003; Salvaterra, Ferrara, \& Schneider 2003). For the particular case we simulated in Paper I, we found that no gas clumps are formed in the dense shell which are Jeans-unstable. This may, however, be because of the still insufficient mass resolution of the simulation. In order to properly address the question of the shell fragmentation and the formation of proto-stellar clouds, simulations with an extremely high mass resolution are needed. Also, more complex configurations, such as collisions between neighboring blast waves originating in more massive systems, may result in the formation of gravitationally bound fragments. We pursue this possibility further in a forthcoming work (Bromm et al., in preparation).

\subsection{Thomson Optical Depth and Mean Metallicity}

In $\S 2$, we have shown that the production of ionizing photons and heavy elements by massive Population III stars is described by fundamental constants in the case where these stars explode as pair-instability supernovae. Here, we derive an approximate but concise relationship between the evolution of the mean metallicity and the total Thomson optical depth, $\tau_{e}$, as follows.

The differential optical depth to Thomson scattering corresponding to the redshift interval $d z$ is

$$
d \tau_{e}=\sigma_{\mathrm{T}} n_{e}(z) c \frac{d t}{d z} d z
$$

where $\sigma_{\mathrm{T}}=6.65 \times 10^{-25} \mathrm{~cm}^{2}$ is the Thomson scattering cross section, and $n_{e}(z)$ the mean electron number density at $z$. The mean electron number density is given by

$$
n_{e}(z)=n_{e}(0)(1+z)^{3} x_{e}(z),
$$

where $x_{e}$ is the ionization fraction at $z$, and $n_{e}(0)$ the present-day number density of electrons. We obtain the contribution from an interval between $z_{1}$ and $z_{2}\left(z_{1}<z_{2}\right)$,

$$
\begin{aligned}
\left.d \tau_{e}\right|_{z_{1} \rightarrow z_{2}} & =\sigma_{\mathrm{T}} n_{e}(0) x_{e}(z) \frac{c}{H_{0}} \\
& \times \frac{(1+z)^{2}}{\left(\Omega_{\Lambda}+\Omega_{\mathrm{m}}(1+z)^{3}\right)^{1 / 2}} d z \\
& \approx \sigma_{\mathrm{T}} n_{e}(0) x_{e}(z) \frac{c}{H_{0}}\left(\frac{1+z}{\Omega_{\mathrm{m}}}\right)^{1 / 2}
\end{aligned}
$$

We have assumed a flat $\Lambda$ CDM model with $H(z)=$ $H_{0}\left[\Omega_{\Lambda}+\Omega_{\mathrm{m}}(1+z)^{3}\right]^{1 / 2}$ and the last approximation holds at high redshifts, certainly for $z>10$.

Neglecting recombination, we can approximate, to the lowest order, the ionization fraction as

$$
x_{e}(z) \propto q(z) .
$$

From equation (10) we obtain for pair-instability SNe,

$$
x_{e}(z) \sim \frac{\bar{Z}(z)}{\zeta_{\mathrm{PISN}} f_{\mathrm{PISN}}} .
$$

Absorbing all the constant factors into a single quantity $\Upsilon$, including $f_{\mathrm{PISN}}$, we express the concise relation as

$$
\int d \tau_{e} \simeq \Upsilon \int_{z} \bar{Z}(z)(1+z)^{1 / 2} d z
$$

Thus, to the lowest order, the evolution of the ionization fraction should be accompanied by the increasing metallicity following equation (21). Integrating equation (21) to $z=z_{\text {chem }}$, when $\bar{Z}=5 \times 10^{-4} Z_{\odot}$, we obtain

$$
\Delta \tau_{\mathrm{e}, \mathrm{PopIII}} \sim 0.06
$$

This is the contribution from early Population III stars expected in our model.

\subsection{Metallicity in the Lyman- $\alpha$ Forest at $z \sim 3-5$}

Metals produced by the early Population III stars should exist ubiquitously in various systems at low redshift. Miralda-Escudé \& Rees (1997) argue that the observed metallicity in the Lyman- $\alpha$ forest at $z \sim 3$ is consistent with a simple picture in which the metals originate from an early generation of stars. The average metallicity $\bar{Z}=10^{-2} Z_{\odot}$ in the Lyman- $\alpha$ forest at $z \sim 3$ is still much higher than the mean metallicity achieved at the end of the Population III era, $z \sim 15$, whereas Songaila (2001) argues that a minimum universal metallicity of about $3.5 \times 10^{-4} Z_{\odot}$ is found in $z \sim 5$ Lyman- $\alpha$ clouds. Remarkably, this is very close to $\bar{Z} \sim 3 \times 10^{-4} Z_{\odot}$ (Figure 1) achieved by the early massive Population III stars. Since the Population III star formation is self-regulating owing to the global radiative feedback and metal enrichment, this value is expected to be approximately the maximum which early Population III stars alone can achieve. Songaila (2001) estimates the mass density of C IV between $z=1.5$ and $z=5$ to be $\Omega_{\mathrm{CIV}}$ IGM $=(2.5-7) \times 10^{-8}$. Assuming $\mathrm{C}$ IV is the dominant ionization stage with ionization fraction of 0.5 (Songaila 1997), the mass density in carbon is $\sim(5-14) \times 10^{-8}$. For a very massive zerometalicity star, the carbon yield is $\sim 4 M_{\odot}$ and nearly constant for the relevant core mass (Heger \& Woosley 2002). From this we obtain the mass density in carbon (more precisely, ${ }^{12} \mathrm{C}$ ) for our model with $f_{\mathrm{PISN}}=1$,

$$
\Omega_{\mathrm{C}_{\mathrm{IV}}, \mathrm{PopIII}} \approx 10^{-8}
$$

at $z=15$. Although smaller than the value that Songaila (2001) obtains for the diffuse IGM at $z \sim 5$, this is not negligible. Indeed, a fraction of the carbon, up to $20 \%$, in the IGM may originate from the early generation Population III stars.

At $z \sim 3$, the bulk of the metals found in the Lyman$\alpha$ forest may also come from ordinary stellar populations formed in galaxies at lower redshift, $z<5$ (e.g., Adelberger et al. 2003). Interestingly, the metallicity evolution computed by Hernquist \& Springel (2003) based on their analytic model of star-formation in galaxies predicts that the average metallicity reaches $\sim 10^{-2} Z_{\odot}$ at $z \sim 3-5$, in good agreement with the observed value in the Lyman- $\alpha$ forest.

\subsection{Enrichment of the Intracluster Medium}

X-ray observations of galaxy clusters reveal that the ICM is enriched to a significant fraction of the solar metallicity (e.g., Mushotzky et al. 1996; White 2000). In fact, a large fraction ( 75\%) of a typical cluster's endowment with heavy elements exists in the ICM rather than in cluster galaxies (Renzini 1997). Clusters retain 
all the metals produced in them, and the determination of the abundances for several elements in the ICM is relatively straightforward, without involving complicated modeling. Thus, the elemental abundances in the ICM offer a clean template against which any theoretical models can be tested. Loewenstein (2001) suggests that preenrichment by a generation of massive Population III stars may account for the abundance anomalies of oxygen, silicon and iron, observed in the ICM. The recent analysis of the relative overabundance of $\mathrm{Si}$ with respect to $\mathrm{Fe}$ in the ICM also indicates the possible contribution from massive Population III stars. Baumgartner et al. (2003) argue that the observed relative overabundance of $\mathrm{Si}$ can be explained by Population III progenitors with a $70 M_{\odot} \mathrm{He}$ core. While including the contribution from massive Population III stars is attractive, the required abundance appears very large. Baumgartner et al. (2003) estimate that the total mass in Population III stars should be at least $\Omega_{\text {PopIII }} \sim 10^{-5}$, in units of the cosmic density parameter, whereas our model predicts only

$$
\Omega_{\text {PopIII }} \sim 5 \times 10^{-7},
$$

at $z=15$. Baumgartner et al. (2003) argue that primordial star formation is enhanced in overdense regions which later become clusters. However, while this might seem plausible, the strong self-regulation of early star formation primarily tends to shift star formation to earlier epochs in overdense regions, rather than locally enhancing the total amount of formed stars. We therefore conclude that metals originating from the earliest generation stars cannot be responsible for the observed abundance anomalies in the ICM.

\subsection{Dust Formation and $\mathrm{H}_{2}$ Production}

We have computed the mean metallicity of the IGM assuming implicitly that all the metals produced and ejected by SN explosions will remain in the gas phase. This is not strictly true; a fraction of the metals will be in the form of dust and hence do not directly affect the gas chemical properties (see however the effect of promoted molecular hydrogen formation discussed later in this section). Schneider, Ferrara, \& Salvaterra (2003a) and Nozawa et al. (2003) calculate the dust depletion factor for pairinstability SNe to be up to 30-40\%. Nozawa et al. (2003) also conclude that the dust formation rate and the relative abundances of the dust grains of various compounds depend sensitively on the mass of the progenitor and on the mixing efficiency in the ejecta. Another complexity is that the produced dust grains are destroyed by thermal sputtering with ions and electrons, and by grain-grain collisions in post-shock regions (Spitzer 1978). Most important, the reverse shock formed in the early stages of a SN explosion can destroy a significant fraction of the dust grains produced in the ejecta (McKee 1989). Unfortunately, the efficiency of dust destruction by shocks is not well known and we need to resort to a simple parametrization. If, for example, the destruction efficiency is very low and nearly all the formed dust grains survive, are dispersed and remain in the IGM, the cumulative amount of dust could be significant. We choose the dust depletion factor to be $f_{\mathrm{dpl}}=0.4$ (Schneider et al. 2003a), and assume a low destruction efficiency, $e_{\mathrm{dest}}=0.1$. Using the global formation rate for Population III stars in equation (13) and assuming $f_{\mathrm{PISN}}=1$, we calculate a conservative upper limit for the mass density of dust in the IGM,

$$
\Omega_{\mathrm{dust}}^{\mathrm{IGM}} \approx 10^{-7}
$$

by $z=15$. This is much smaller than, for example, the density of intergalactic dust, $\Omega_{\mathrm{dust}}^{\mathrm{IGM}} \sim 10^{-4-5}$ at $z=0.5$ (Aguirre 1999; see also Pei, Fall, \& Hauser 1995; Croft et al. 2000), and thus appears to make negligible contribution to the dust in the diffuse IGM even if the grains survive until the present epoch. We point out, however, that even a small quantity of dust in the gas accelerates $\mathrm{H}_{2}$ production and hence affects the efficiency of gas cooling and star formation when the gas metallicity is still low (Hirashita et al. 2002). Todini \& Ferrara (2001) argue that $\mathrm{H}_{2}$ production on dust grains dominates over the usual $\mathrm{H}^{-}$ channel when the dust-to-gas ratio is greater than 5 percent of the local Galactic value $D_{g} \sim 6 \times 10^{-3}$. Our model predicts that the mean dust-to-gas ratio is $\sim 3 \times 10^{-6}$ at $z=15$ for $f_{\mathrm{dpl}}=0.4$ and $e_{\mathrm{dest}}=0.1$, still an order of magnitude smaller than the critical value. $\mathrm{H}_{2}$ production on dust grains could still be important if the dust-to-gas ratio were locally high, either because the distribution is inhomogeneous, or because the dust grains remain preferentially within a confined region around the SN site.

\subsection{Building-up an Early X-ray Background}

It has been suggested that X-rays could act as a source of positive feedback by boosting the free electron fraction in distant regions. This would in turn promote the formation of $\mathrm{H}_{2}$, hence enabling enhanced cooling of primordial gas (e.g., Haiman, Rees, \& Loeb 1996; Oh 2001; Glover \& Brand 2003). Apart from early quasars (e.g., Eisenstein \& Loeb 1995; Bromm \& Loeb 2003; Madau et al. 2003), SNRs are the only plausible X-ray sources at very high redshifts. High redshift SNRs cool via inverse-Compton cooling, losing a large fraction of their energy to CMB photons (Oh, Cooray, \& Kamionkowski 2003). Some fraction of the thermal energy is also converted into X-rays via thermal bremsstrahlung, and yet another unknown fraction goes into relativistic electrons which could emit $\mathrm{X}$-rays via inverse-Compton scattering of CMB photons. Unfortunately, the relative fractions of energy delivered to these processes is highly uncertain, and likely to be timedependent during the evolution of the SNRs. In order to obtain a rough order-of-magnitude estimate, we calculate the evolution of an early X-ray background using some working assumptions as follows. We consider only $\mathrm{X}$-rays with energies greater than $1 \mathrm{keV}$, because the IGM is nearly optically thin to these X-rays, and assume further that a fraction $c_{\mathrm{X}}$ of the total $\mathrm{SN}$ explosion energy is converted to radiation in the relevant X-ray band, between $1 \mathrm{keV}$ and $5 \mathrm{keV}$. We set, somewhat arbitrarily, $c_{\mathrm{X}}=0.1$ as our fiducial value. Note that this value is significantly larger than the one assumed by Glover \& Brand (2003) as a maximum efficiency case, based on the estimate of Terlevich et al. (1992) for X-ray bright remnants in dense environments, $c_{\mathrm{X}} \approx 0.01$. Hence, the results presented below provide a conservative upper limit.

For the emissivity of the SNRs, we rather crudely model it to be described by a power law $L_{\nu} \propto \nu^{-1}$ in the narrow (1-5 keV) energy range. By noting that radiative cooling 
in the SNRs occurs mainly in the first few million years for the original SN energy of $\sim 10^{53}$ ergs (see section 2.3), the time-averaged luminosity in X-rays from a single SNR is estimated to be

$$
L_{\nu} \approx 4.2 \times 10^{20}\left(\frac{\nu_{0}}{\nu}\right)^{-1} \operatorname{ergs~s}^{-1} \mathrm{~Hz}^{-1},
$$

where $h \nu_{0}=1 \mathrm{keV}$. We compute the evolution of the diffuse $\mathrm{X}$-ray flux by solving the cosmological radiative transfer equation (e.g., Peebles 1993)

$$
\left(\frac{\partial}{\partial t}-\nu H(z) \frac{\partial}{\partial \nu}\right) J=-3 H(z) J+\frac{c}{4 \pi} \epsilon
$$

where $J$ is the specific intensity in units of ergs s $\mathrm{s}^{-1} \mathrm{~cm}^{-2}$ $\mathrm{Hz}^{-1} \mathrm{sr}^{-1}, H(z)$ the Hubble parameter at redshift $z, c$ the speed of light, and $\epsilon$ the proper volume-averaged emissivity. We use this unconventional unit for the X-ray flux in order to facilitate comparisons with the commonly considered soft-UV background radiation. We compute the volume emissivity by combining equation (26) with our model SFR (equation [13], see also Figure 1), assuming that all the Population III stars die as pair-instability $\mathrm{SNe}$ $\left(f_{\mathrm{PISN}}=1\right)$. This simple model again serves as a maximal case in terms of X-ray emission efficiency.

Figure 3 shows the evolution of the X-ray background radiation intensity at $1 \mathrm{keV}$. It is clear that the flux reaches only up to $10^{-24} \operatorname{ergs~s}^{-1} \mathrm{~cm}^{-2} \mathrm{~Hz}^{-1} \mathrm{sr}^{-1}$ by $z=15$. Machacek, Bryan, \& Abel (2003) performed numerical simulations of pre-galactic object formation to examine the effect of the positive feedback from a diffuse X-ray background in the early universe. They conclude that the net effect is quite small for X-ray fluxes below $\sim 10^{-23}$ ergs s $\mathrm{s}^{-1} \mathrm{~cm}^{-2} \mathrm{~Hz}^{-1} \mathrm{sr}^{-1}$. To result in a positive feedback that counteracts the negative feedback due to photodissociating radiation, an order of magnitude higher flux is necessary, which is two orders of magnitude higher than our estimate for the X-ray flux at $z=15$. Overall, the effect of the early X-ray background caused by SNRs to the primordial gas cooling appears unimportant at $z>15$.

\subsection{The G-dwarf Problem}

It has often been suggested that the paucity of metalpoor stars in the solar neighborhood relative to the prediction of theoretical models, the so-called G-dwarf problem, can be resolved by invoking early massive Population III stars (Cayrel 1986; see also Larson 1998 for a recent review). In fact, the kind of pre-enrichment by early generation stars proposed here may offer an excellent resolution if the metallicity of the gas is sufficiently high initially. The simplest models require a significant degree of preenrichment in the Galactic disk (e.g., Binney \& Tremaine $1987), Z_{\text {init }}=0.25 Z_{\odot}$, whereas our model predicts far too small a metallicity accomplished by Population III stars alone, $\sim 10^{-4} Z_{\odot}$. Thus, considering the self-regulating nature of early star formation (see $\S 3.1$ ), we conclude that massive Population III stars do little to resolve the classical G-dwarf problem.

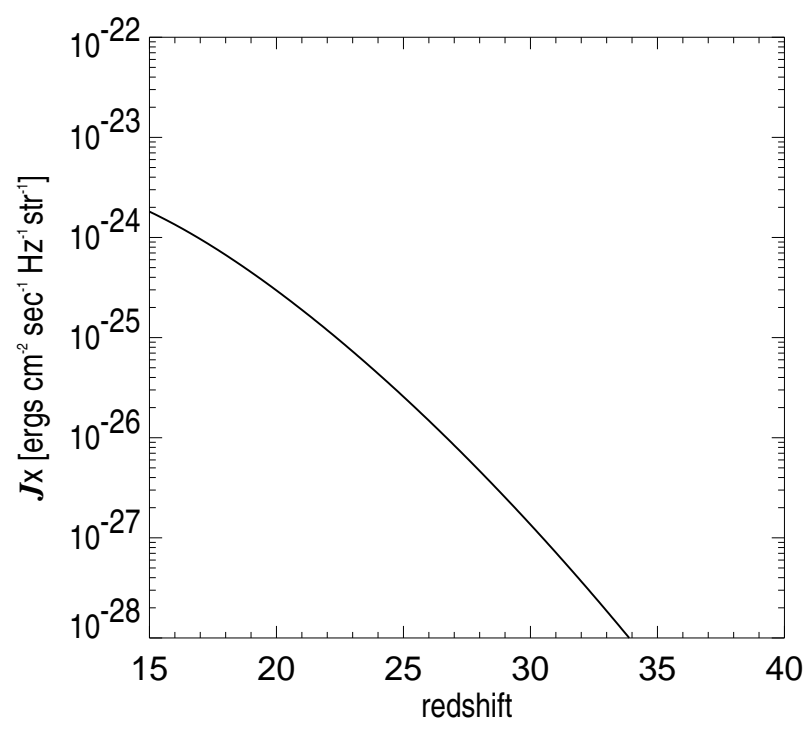

FIG. 3.- The evolution of the X-ray background radiation intensity. We use unconventional units, ergs $\mathrm{cm}^{2} \mathrm{~s}^{-1} \mathrm{~Hz}^{-1} \mathrm{sr}^{-1}$, in order to compare with the typical intensity of the soft-UV background radiation $J_{\mathrm{UV}} \sim 10^{-21-23}$ (in the above units), which is responsible for the negative feedback on $\mathrm{H}_{2}$ cooling.

However, massive Population III stars may account for a modern version of this problem - the paucity of extremely metal-poor stars in the Galactic halo. As we have shown in previous sections, a prompt enrichment to $\sim 10^{-4} Z_{\odot}$ is achieved by $z=15$. In fact, the gas metallicity around the SN sites, where most plausibly the second generation stars will be formed, is higher than this mean value, and thus, within our model, the second generation stars are expected to have metallicities at or above this critical value. Observationally, with the important exception of the ultra-metal poor star discovered by Christlieb et al. (2002), the paucity of stars with $[\mathrm{Fe} / \mathrm{H}]<-4$ is apparent in our Galaxy. It would be extremely important to derive the abundance pattern of such stars through a systematic search (e.g., Christlieb 2003).

\subsection{Running Spectral Index Models}

The rapid metal-enrichment by massive Population III stars has important implications for some variant CDM models. Based on a joint analysis of the first-year WMAP data, the $2 \mathrm{dF}$ galaxy redshift survey, and the Lyman- $\alpha$ forest, Spergel et al. (2003) conclude that cosmological models with a running primordial power spectrum are favored over power-law models with $n \approx 1$ (see also Peiris et al. 2003). However, post- WMAP studies indicate that the observed high optical depth is not achieved in such models with a strong running, because the formation of pre-galactic size objects is systematically delayed owing to the suppressed power on small scales (e.g., Somerville et al. 2003). Chiu, Fan, \& Ostriker (2003) and Avelino \& Liddle (2003) indeed showed that strong running of the primordial power results in considerable delay of reionization. Yoshida et al. (2003b) carried out cosmological hydrodynamic simulations of early structure formation and concluded that the running spectral index (RSI) model is incompatible with the observed high Thomson optical depth. However, Yoshida et al. also argue that the resulting Thomson optical depth can be made marginally con- 
sistent with the WMAP data, if the ionizing photon production rate is extremely high in galaxies at $z>6$. This practically indicates that, in the RSI model, a top-heavy IMF for the stars formed in galaxies must be maintained over a long period, $6<z<20$.

The problematic nature of this requirement can be appreciated by noting that the total elapsed time to $z=6$ in a $\Lambda \mathrm{CDM}$ cosmology is approximately $10^{9}$ years. Very massive stars, like those considered here, have lifetimes $\sim 2 \times 10^{6}$ years. Thus, if star formation commences at high redshifts, $z \gtrsim 20$, nearly 500 generations of these high-mass stars could have formed. As we indicate below, the metals from these stars must be "hidden" to an improbably high degree of precision for Population III star formation not to self-terminate well before $z=6$.

Here, we show that, in scenarios such as the running spectral index model, a large quantity of metals is necessarily produced if it is assumed that Population III star formation continues until $z=6$. Let us consider a simplified model in which the stellar IMF is essentially a $\delta$ function at $300 M_{\odot}$, i.e., all the stellar baryons are converted into very massive stars. This is the most efficient way of getting ionizing photons out of a certain mass of baryons converted into stars, because the luminosity per stellar mass is essentially a constant for metal-free stars with mass exceeding $300 M_{\odot}$. In this case we may simply assume a high ionizing photon production rate per stellar mass of $1.6 \times 10^{48} / M_{\odot}$. As in Yoshida et al. (2003b), the star-formation rate density for the RSI model is computed using the same functional fit (equation [13) with $p=1.5, \tilde{q}=3.05, r=1.85, \tilde{\alpha}=0.01, \beta=0.057$, and $\dot{\rho}_{*}(0)=0.0176 M_{\odot} \mathrm{yr}^{-1} \mathrm{Mpc}^{-3}$.
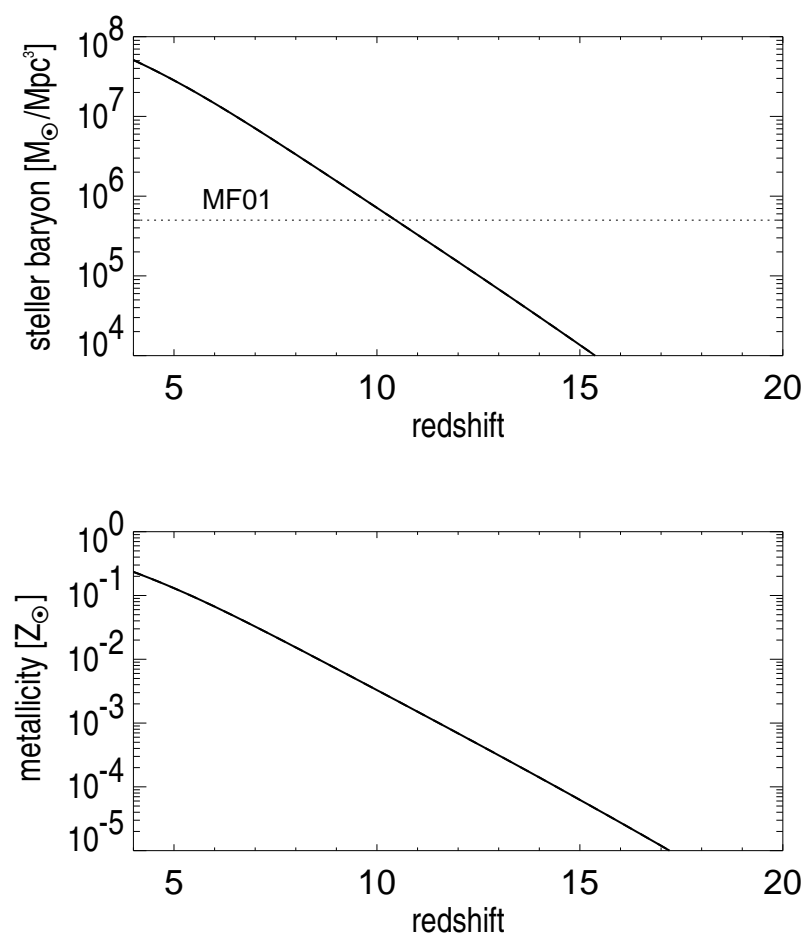

Fig. 4.- We plot the cumulative mass of stellar baryons, which is locked up in black holes in our extreme model (top), and the mass-weighted mean metallicity (bottom) for the RSI model on the assumption that all the stellar baryons are processed through very massive stars.

Figure 4 shows the cumulative mass density of stellar baryons. It directly corresponds to the mass density of black holes if we assume that all the stars become $\mathrm{BHs}$, i.e., their masses are greater than $\sim 300 M_{\odot}$. In the plot, the dotted line indicates the present-day mass density in black holes in our local universe estimated by Merritt \& Ferrarese (2001). Clearly the mass density increases beyond the present-day mass density by $z=10$. On the other hand, if all the stars are massive, but within the pair-instability SN range of $140 M_{\odot}<M<260 M_{\odot}$, up to half of the stellar mass can be processed into heavy elements and expelled. Clearly these models are idealized, but they allow us to explore the possible parameter space. On these assumptions and using the SFR for the RSI model, we compute the mass-weighted mean metallicity. The bottom panel of Figure 4 shows the evolution of the mean metallicity $\bar{Z}$. Remarkably, the critical metallicity $\sim 10^{-4} Z_{\odot}$ is achieved already by $z \sim 14$ and it reaches $\sim 10^{-1} Z_{\odot}$ by $z=5$. It is now clear that, if we resort to the extremely top-heavy IMF in the RSI model to produce enough UV photons to reionize the universe early on, the total amount of heavy elements quickly rises up to a point where the gas chemical properties are expected to change (Bromm et al. 2001b). It is conceivable that all the massive stars collapse to black holes so that the IGM remains chemically pristine without metal-pollution, but then perhaps too much baryonic mass goes into black holes, which appears to be incompatible with the recent estimate of the local mass density in black holes. In summary, a running of the primordial power spectrum seems to be incompatible with various observations in one way or another. A similar conclusion is expected to obtain for other variant CDM models, such as those involving warm dark matter (e.g., Somerville et al. 2003; Yoshida et al. 2003c).

\section{SUMMARY}

We have revisited the cosmological consequences of an early generation of Population III stars. We have primarily considered very massive $\left(\sim 100-300 M_{\odot}\right)$ stars and used a detailed model of the global star formation rate which is derived from semi-analytic modeling and the results of cosmological hydrodynamic simulations that include radiative feedback effects. We have shown that the first stars alone can produce a sufficient number of photons to reionize a significant volume of the IGM by $z \sim 15$, if the stars are very massive, in agreement with cosmological radiative transfer calculations (e.g., Sokasian et al. 2003a). Furthermore, on the assumption that the majority of the stars explode as pair-instability $\mathrm{SNe}$, the mean IGM metallicity increases to the critical value above which the gas cooling efficiency is greatly enhanced. This may lead to the formation of ordinary stellar populations including low-mass stars (e.g. Mackey et al. 2003). We also discussed possible implications of the results. Although a broad range of observations indicates that the kind of massive stars we considered in the present paper existed in the early universe, the simple model may not be able to explain the observations in details. Clearly, further studies using more sophisticated models are necessary to examine the cosmological consequences of early star formation. In particular, we will explore the contribution from the second (and subsequent) generation stars forming at $z<13$ in future work.

The evidence for an early generation of very massive 
stars may well be strengthened by future observations. The ongoing operation of $W M A P$ will yield a more precise value for the total optical depth to reionization. In the longer term, post- WMAP CMB polarization experiments such as Planck will probe the reionization history (e.g., Kaplinghat et al. 2003). Detection of the secondorder polarization anisotropies on arcminute scales can place strong constraint on the details of reionization (e.g., Liu et al. 2001). Near-infrared observations of afterglows from high-redshift gamma-ray bursts can also be used to probe the reionization history at possibly $z>10$ (Barkana \& Loeb 2003; Inoue et al. 2003; Yoshida \& Bloom 2003, in preparation). Mapping the morphological evolution of reionization may be possible by observations of redshifted $21 \mathrm{~cm}$ emission by the Square Kilometer Array and LOFAR (e.g., Tozzi et al. 2000; Furlanetto, Sokasian, \& Hernquist 2004). Data from these future observations will provide a more complete picture of cosmic reionization and will enable us to distinguish the sources responsible for reionization. The precise measurement of the near-IR cosmic background radiation will constrain the total amount of light from early generation stars (Santos, Bromm, \& Kamionkowski 2002; Salvaterra \& Ferrara 2003). Ultimately, direct imaging and spectroscopic observations of high redshift star clusters by the James Webb Space Telescope will probe the evolution of stellar populations up to $z \sim 10-15$ (e.g., Stiavelli, Fall, \& Panagia 2003; Tumlinson, Shull, \& Venkatesan 2003).
Finally, measurements of the relative abundances of various heavy elements in metal-poor stars should provide valuable information on the chemical history of the universe (e.g., Burris et al. 2000; Norris et al. 2002). Interestingly, a strong argument against very massive $\left(>140 M_{\odot}\right)$ stars comes from the observed abundance pattern of $\mathrm{C}$ rich, extremely Fe-deficient stars (Christlieb et al. 2002; Umeda \& Nomoto 2003; but see Schneider et al. 2003b). It remains to be seen whether or not such stars are truly second generation stars and their elemental abundances should precisely reflect the metal-yield from the first SNe. Observations of a large number of extremely metal-poor stars will construct better statistics (Norris, Ryan, \& Beers 2001) and improve constraints on any models for the early chemical evolution. Understanding the origin of the first heavy elements in the universe and the nature of the sources that are responsible for cosmic reionization will require the concerted use of data from these broad classes of observations.

We thank Evan Scannapieco for helpful comments, and Aaron Sokasian for providing us with the SFR data. NY acknowledges support from the Japan Society of Promotion of Science Special Research Fellowship (grant 02674). This work was supported in part by NSF grants ACI 9619019, AST 98-02568, AST 99-00877, and AST 00-71019 and NASA ATP grant NAG5-12140.

\section{REFERENCES}

Abel, T., Anninos, P., Norman, M. L., \& Zhang, Y. 1998, ApJ, 508, 518

Abel, T., Bryan, G. L., \& Norman, M. L. 2002, Science, 295, 93

Adelberger, K. L., Steidel, C. C., Shapley, A. E., \& Pettini, M. 2003, ApJ, 584, 45

Aguirre, A. 1999, ApJ, 512, L19

Aguirre, A., Hernquist, L., Schaye, J., Weinberg, D., Katz, N., \& Gardner, J. 2001a, ApJ, 560, 599

Aguirre, A., Hernquist, L., Schaye, J., Katz, N., Weinberg, D., \& Gardner, J. 2001b, ApJ, 561, 521

Avelino, P. P. \& Liddle, A. R. 2003, MNRAS, submitted (astro-ph/0305357)

Barkana, K., \& Loeb, A. 2003, ApJ, in press (astro-ph/0305470

Barkat, Z., Rakavy, G., \& Sack, N. 1967, Phys. Rev. Lett., 18, 379

Baraffe, I., Heger, A., \& Woosley, S. E. 2001, ApJ, 550, 890

Baumgartner, W. H., Loewenstein. M.. Horner. D. J., \& Mushotzky, R. F. 2003, ApJ, submitted (astro-ph/0309166

Bertschinger, E. 1983, ApJ, 268, 17

Binney, J. \& Tremaine, S. 1987, Galactic Dynamics (Princeton University Press)

Bond, J. R., Arnett, W. D., \& Carr, B. J. 1984, ApJ, 280, 825

Bromm, V., Coppi, P. S., \& Larson, R. B. 1999, ApJ, 527, L5

Bromm, V., Coppi, P. S., \& Larson, R. B. 2002, ApJ, 564, 23

Bromm, V., Ferrara, A., Coppi, P. S., \& Larson, R. B. 2001a, MNRAS, 328, 969

Bromm, V., Kudritzki, R. P., \& Loeb, A. 2001b, ApJ, 552, 464

Bromm, V., \& Loeb, A. 2003, ApJ, 596, 34

Bromm, V., Yoshida, N., \& Hernquist, L. 2003, ApJ, 596, L135 (Paper I)

Burris, D. L., Pilachowski, C. A., Armandroff, T. E., \& Sneden, C. 2000, ApJ, 544, 302

Cayrel, R. 1986, A\&A, 168, 81

Cen, R. 2003, ApJ, 591, 12

Chiu, W. A., Fan, X., \& Ostriker, J. P. 2003, ApJ, 599, 759

Christlieb, N., et al. 2002, Nature, 419, 904

Christlieb, N. 2003, Revs. Mod. Astronomy, 16, 191

Couchman, H. M. P., \& Rees, M. J. 1986, MNRAS, 221, 53

Croft, R.A.C., Davé, R., Hernquist, L., \& Katz, N. 2000, ApJ, 534, 123

Dekel, A., \& Silk, J. 1986, ApJ, 303, 39

Di Matteo, T., Croft, R.A.C., Springel, V., \& Hernquist, L. 2003a, ApJ, 593, 56

Di Matteo, T., Croft, R.A.C., Springel, V., \& Hernquist, L. 2003b, ApJ, submitted (astro-ph/0309533)
Dopita, M. A., Mathewson, D. S., \& Ford, V. L. 1985, ApJ, 297, 599

Efremov, Y. N., \& Elmegreen, B. G. 1998, MNRAS, 299, 643

Eisenstein, D. J., \& Loeb, A. 1995, ApJ, 443, 11

Ferrara, A. 1998, ApJ, 499, L17

Freudling, W., Corbin, M. R., \& Korista, K. T. 2003, ApJ, 587, L67 Fryer, C. L., Woosley, S. E., \& Heger, A. 2001, ApJ, 550, 372

Furlanetto, S. R., \& Loeb, A. 2003, ApJ, 588, 18

Furlanetto, S. R., Sokasian, A., \& Hernquist, L. 2004, MNRAS, 347, 187

Glover, S. C. O., \& Brand, P. W. J. L. 2003, MNRAS, 340, 210

Haiman, Z., Abel, T., \& Madau, P. 1999, ApJ, 551, 599

Haiman, Z., \& Holder, G. P. 2003, ApJ, 595, 1

Haiman, Z., Rees, M. J., \& Loeb, A. 1996, ApJ, 467, 522

Heger, A., \& Woosley, S. E. 2002, ApJ, 567, 532

Heger, A., Fryer, C. L., Woosley, S. E., Langer, N., \& Hartmann D. H. 2003, ApJ, 591, 288

Hernquist, L., \& Springel, V. 2003, MNRAS, 341, 1253

Hirashita, H., Hunt, L. K., \& Ferrara, A. 2002, MNRAS, 330, L19

Inoue. A. K.. Yamazaki, R., \& Nakamura, T. 2003, ApJ, submitted (astro-ph/0308206)

Islam, K. K., Taylor, J. E., \& Silk, J. 2003, MNRAS, 340, 647

Kaplinghat, M., Chu, M., Haiman, Z., Holder, G., \& Knox, L. 2003, ApJ, 583, 24

Kogut, A., et al. 2003, ApJS, 148, 161

Larson, R. B. 1974, MNRAS, 169, 229

Larson, R. B. 1998, MNRAS, 301, 569

Liu, G.-C., Sugiyama, N., Benson, A. J., Lacey, C. G., \& Nusser, A. 2001, ApJ, 504, 516

Loewenstein, M. 2001, ApJ, 557, 576

Machacek, M. E., Bryan, G. L., \& Abel, T. 2003, MNRAS, 338, 273

Mackey, J., Bromm, V., \& Hernquist, L. 2003, ApJ, 586, 1

Mac Low, M.-M., \& Ferrara, A. 1999, ApJ, 513, 142

Madau, P., Ferrara, A., \& Rees, M. J. 2001, ApJ, 555, 92

Madau, P., \& Rees, M. J. 2001, ApJ, 551, L27

Madau, P., Rees, M. J.. Volonteri. M., Haardt, F., \& Oh, S. P. 2003,

ApJ, submitted (astro-ph/0310223)

McCray, R., \& Katatos, M. 1987, ApJ, 317, 190

McKee, C., 1989, in Interstellar Dust: Proceedings of the 135th Symposium of the International Astronomical Union, eds. L. J. Allamandola and A. G. G. M. Tielens. IAUS no. 135, Kluwer Academic Publishers (Dordrecht), 431

Merritt, D., \& Ferrarese, L. 2001, MNRAS, L30

Miralda-Escudé, J., \& Rees, M. J. 1997, ApJ, 478, L57 
Mori, M., Ferrara, A., \& Madau. P. 2002, ApJ, 571, 40

Mushotzky, R. F., et al. 1996, ApJ, 466, 686

Nakamura, F., \& Umemura, M. 2001, ApJ, 548, 19

Nakamura, F., \& Umemura, M. 2002, ApJ, 569, 549

Nakasato, N., \& Shigeyama, T. 2000, ApJ, 541, 59

Norris, J. E., Ryan, S. G., \& Beers, T. C. 2001, ApJ, 561, 1034

Norris, J. E., Ryan, S. G., Beers, T. C., Aoki, W., \& Ando, H. 2002, ApJ, 569, L107

Nozawa, T., Kozasa, T., Umeda, H., Maeda, K., \& Nomoto, K. 2003, ApJ, 598, 785

Oh, S. P. 2001, ApJ, 569, 558

Oh, S. P., Cooray, A., \& Kamionkowski, M. 2003, MNRAS, 342, 20

Oh, S. P., \& Haiman, Z. 2002, ApJ, 569, 558

Oh, S. P., Nolett, K. M., Madau, P., \& Wasserburg, G. J. 2001, ApJ, $562, \mathrm{~L} 1$

Omukai, K., \& Palla, F. 2003, ApJ, 589, 677

Omukai, K., \& Yoshii, Y. 2003, ApJ, 599, 7460

Ostriker, J. P., \& Gnedin, N. 1996, ApJ, 472, L66

Ostriker, J. P., \& Cowie, L. L. 1981, ApJL, 243, 127

Ostriker, J. P., \& McKee, C. F. 1988, Rev. Mod. Phys., 60, 1

Peebles, P. J. E. 1993, Principles of Physical Cosmology (Princeton: Princeton Univ. Press)

Pei, Y., Fall, M., \& Hauser, M. 1999, ApJ, 522, 604

Peiris, H. V., et al. 2003, ApJS, 148, 176

Pettini, M., Madau, P., Bolte, M., Prochaska, J. X., Ellison, S. L., \& Fan, X. 2003, ApJ, 594, 695

Prochaska, J. X. 2002, ApJ, 582, 49

Qian, Y.-Z., Sargent, W. L. W., \& Wasserburg, G. J. 2002, ApJ, 569, L61

Qian, Y.-Z., \& Wasserburg, G. J. 2001, ApJ, 549, 337

Qian, Y.-Z., \& Wasserburg, G. J. 2002, ApJ, 567, 515

Renzini, A. 1997, ApJ, 488, 35

Ricotti, M., Gnedin, N. Y., \& Shull, J. M. 2002, ApJ, 575, 33

Salvaterra, R., \& Ferrara, A. 2003, MNRAS, 339, 973

Salvaterra, R.. Ferrara. A..\& Schneider, R. 2003, MNRAS, submitted (astro-ph/0304074)

Santos, M. K., Bromm, V., \& Kamionkowski, M. 2002, MNRAS, 336,1082

Scannapieco, E., Schneider, R., \& Ferrara, A. 2003, ApJ, 589, 35

Scannapieco, E., Thacker, R. J., \& Broadhurst, T. J. 2001, ApJ, 557,605

Schaerer, D. 2002, A\&A, 382, 28

Schaerer, D. 2003, A\&A, 397, 527

Schaye, J., Theuns, T., Rauch, M., Efstathiou, G., \& Sargent, W. L. W. 2000, MNRAS, 318, 817

Schaye, J., Aguirre, A., Kim, T.-S., Theuns, T., Rauch, M., \& Sargent, W. L. W. 2003, ApJ, 596, 768

Schneider, R., Ferrara, A., \& Salvaterra, R. 2003a, MNRAS, submitted (astro-ph/0307087)

Schneider, R., Ferrara, A., Salvaterra, R., Omukai, K., \& Bromm, V. 2003b, Nature, 422, 869
Schneider, R., Natarajan, P., Ferrara, A.,\& Omukai, K. 2002, ApJ, 571,30

Sedov, L. I. 1959, Similarity and Dimensional Methods in Mechanics (New York: Academic Press)

Shapiro, P. R., Iliev, I. T., \& Raga, A. C. 2003, MNRAS, submitted (astro-ph/0307266

Sokasıan, A., Yoshıda, N., Abel. T.. Hernquist, L., \& Springel, V. 2003a, MNRAS, in press, (astro-ph/0307451)

Sokasian, A., Abel, T., Hernquist, L., \& Springel, V. 2003b, MNRAS, 334, 607

Somerville, R.S., Bullock, J.S., \& Livio, M. 2003, ApJ, 593, 616

Songaila, A. 1997, ApJ, 490, L1

Songaila, A. 2001, ApJ, 561, L153 (erratum 568, L139 [2002])

Spergel, D.N., et al. 2003, ApJS, 148, 175

Springel, V., \& Hernquist, L. 2003a, MNRAS, 339, 289

Springel, V., \& Hernquist, L. 2003b, MNRAS, 339, 312

Spitzer, L. 1978, Physical Processes in the Interstellar Medium (New York: Wiley)

Stiavelli. M.. Fall. S. M., \& Panagia, N. 2003, ApJ, in press (astro-ph/0309835)

Tegmark, M., Silk, J., Rees, M. J., Blanchard, A., Abel, T., \& Palla, F. 1997, ApJ, 474, 1

Terlevich, R., Tenorio-Tagle, G., Franco, J., \& Melnick, J. 1992, MNRAS, 255, 713

Thacker, R. J., Scannapieco, E., \& Davis, M. 2002, ApJ, 581, 836

Todini, P., \& Ferrara, A. 2001, MNRAS, 325, 726

Tozzi, P., Madau, P., Meiksin, A., \& Rees, M. J. 2000, ApJ, 528, 597

Tumlinson, J., Shull, J. M., \& Venkatesan, A. 2003, ApJ, 584, 608

Umeda, H., \& Nomoto, K. 2002, ApJ, 565, 385

Umeda, H., \& Nomoto, K. 2003, Nature, 422, 871

Vader, J. P. 1986, ApJ, 305, 669

Volonteri, M., Haardt, F., \& Madau, P. 2003, ApJ, 582, 559

Wada, K., \& Venkatesan, A. 2003, ApJ, 591, 38

Wandel, A. 1985, ApJ, 294, 385

Whalen, D., Abel, T., \& Norman, M. 2003, ApJ, submitted (astro-ph/0310283)

White, D. A. 2000, MNRAS, 312, 663

Wise, J. H., \& Abel, T. 2003, in AIP Conf. Proc. 666, The Emergence of Cosmic Structure, ed. S. S. Holt \& C. S. Reynolds (Woodbury: AIP), 97

Woosley, S. E., Heger, A., \& Weaver, T. A. 2002, Rev. Mod. Phys., 74,1015

Wyithe, J. S. B., \& Loeb, A. 2003a, ApJ, 586, 693

Wyithe, J. S. B., \& Loeb, A. 2003b, ApJ, 588, L69

Yoshida, N., Abel, T., Hernquist, L., \& Sugiyama, N. 2003a, ApJ, 592,645

Yoshida, N., Sokasian, A., Hernquist, L., \& Springel, V. 2003b, ApJ, 598, 73

Yoshida, N., Sokasian, A., Hernquist, L., \& Springel, V. 2003c, ApJ, 591, L1 